Physics

Physics Research Publications

\title{
Microscopic origin of magnetoelectric coupling in noncollinear multiferroics
}

J. P. Hu 


\title{
Microscopic Origin of Magnetoelectric Coupling in Noncollinear Multiferroics
}

\author{
Jiangping $\mathrm{Hu}$ \\ Department of Physics, Purdue University, West Lafayette, Indiana 47007, USA
}

(Received 7 September 2007; published 20 February 2008)

\begin{abstract}
An universal microscopic mechanism to understand the interplay between the electric and magnetic degrees of freedom in noncollinear multiferroics is developed. In a system with a strong spin-orbit coupling, we show that there is a pure electric mechanism that the ferroelectricity is generated by noncollinear magnetism through an electric current cancellation process, which saves the pure electric energy. This mechanism provides a simple estimation and sets a physical limitation of the value of ferroelectricity in noncollinear multiferroic materials.
\end{abstract}

DOI: 10.1103/PhysRevLett.100.077202

PACS numbers: 75.80.+q, 75.47.Lx, 77.80.-e

Multiferroics are materials in which magnetic and electric orders are strongly coupled, and have attracted increasing attention [1,2]. Recent experimental research on multiferroics has shown that ferroelectricity and magnetism not only coexist in the same material but also couple so strongly that the magnetic degree of freedom can be manipulated by an electric field and the electric degree of freedom can be manipulated by a magnetic field [3-9]. This property promises important technological applications in the future.

What is the microscopic mechanism that causes the strong coupling between these 2 degrees of freedom? This is the most fundamental problem in understanding the physics of multiferroics. However, due to the complexity of such materials, theoretical understanding of the microscopic mechanism of strong magnetoelectric coupling is quite limited. Microscopically, the only known mechanism to date, is related to the DzyaloshinskiiMoriya (DM) $[10,11]$ interaction $[12,13]$, such as the inverse DM mechanism [12] which is based on the idea that spin currents are induced between the noncollinear spins, and can therefore be considered as electric moments. However, this mechanism is limited in two important ways: (i) it lacks quantitative prediction results; (ii) strictly speaking, the concept of spin current in spin orbital coupled system is not well defined. Most other analytic theoretical works are based on the phenomenological analysis. For example, through symmetric analysis in the Ginzburg-Landau approach to thermodynamics, Mostovoy [14] has shown that the relationship between the ferroelectric order $\vec{P}$ and magnetic order $\vec{M}$ in spiral magnets is given by

$$
\vec{P} \propto \vec{M} \times(\vec{\nabla} \times \vec{M}) .
$$

Equation (1) qualitatively explains experimental results [3]. However, the phenomenological theory is unable to provide the microscopic origins and fix the coupling parameters in Eq. (1).

This Letter proposes a simple but universal new microscopic mechanism to understand the magnetoelectric coupling in noncollinear multiferroics. In a system with a strong spin-orbit coupling, we show that there is a pure electric mechanism that ferroelectricity is induced by noncollinear magnetism through an electric current cancellation process which saves the pure electric energy. In this new mechanism, the ferroelectricity only takes place in the partially filled electron band, which differs from the physics in the conventional ferroelectric materials where the ferroelectricity is generated by the lattice dynamics and the electron bands are either fully occupied or empty. The proposed mechanism can provide quantitative predictions. It explains why the value of ferroelectricity in noncollinear multiferroics is two or three orders smaller than that in the conventional ferroelectric materials. Moreover, the mechanism enables us to consider a unified picture of strong magnetoelectric coupling in multiferroics.

We know from the Maxwell equation that static magnetism and static electricity do not couple with each other, and that dynamic electricity such as an electric current can generate a magnetization. The inverse process also exists, namely, a magnetization can generate an electric current. Let us revisit the definition of the electric current. The current operator of the electron is defined as the change in Hamiltonian with respect to the variation of the vector potential of electromagnetic field, i.e.,

$$
\vec{j}=-c \frac{\delta H}{\delta \vec{A}} .
$$

In nonrelativistic quantum mechanics, the definition of the electric current includes three terms generated from three different physics: (i) the contribution of standard momentum, (ii) the spin contribution illustrated in standard quantum textbooks $[15,16]$, (iii) the contribution of spin orbital coupling. To be more specific, we consider a single electron in a band structure. The electron is described by the Hamiltonian

$$
H_{e}=\frac{\left(\vec{p}-e \frac{\vec{A}}{c}\right)^{2}}{2 m^{*}}+\alpha\left(\vec{p}-e \frac{\vec{A}}{c}\right) \cdot[\vec{\sigma} \times \vec{\nabla} V(\vec{r})]-\mu(\vec{\nabla} \times \vec{A}) \cdot \vec{\sigma}
$$

where, $m^{*}$ is the effective mass of electrons, $\alpha$ is the 
effective spin orbital coupling parameter, $\mu=\frac{g e}{2 m c}$ and $\vec{\sigma}$ is the spin of the electron. In the absence of the external electrodynamic field, i.e. $\vec{A}=0$, for a given wave function, $\Psi(\vec{r})$, the electric current derived from Eq. (2) is given by

$$
\vec{j}=\vec{j}_{0}+\mu c \vec{\nabla} \times\left(\Psi^{*} \vec{\sigma} \Psi\right)+\alpha e\left(\Psi^{*} \vec{\sigma} \Psi\right) \times \vec{\nabla} V(\vec{r}),
$$

where

$$
\vec{j}_{0}=\frac{i e \hbar}{2 m^{*}}\left[\left(\vec{\nabla} \Psi^{*}\right) \Psi-\Psi^{*}(\vec{\nabla} \Psi)\right] .
$$

The three terms in Eq. (4) precisely correspond to the three physics discussed above. The first term usually dominates over the other two terms in transport. Therefore, the last two terms are usually ignored and are not familiar to most people. Here we show that in multiferroics which are insulators, the interplay between the last two terms in Eq. (4) provides a fundamental mechanism to understand the magnetoelectric coupling.

In the noncollinear multiferroics, $R \mathrm{MnO}_{3}, \quad R=$ Dy, Tb, Gd ..., a noncollinear spiral magnetic order has been observed [3]. The spiral magnetic order is formed by the localized spins of $\mathrm{Mn}$ atoms. To study the electronic physics, we can use a Kondo-lattice type of model. We consider that the electrons in the band couple to the localized spins of the Mn atoms through spin exchange coupling. Through the exchange coupling, we can naturally assume that the magnetic ordering of the localized spins also generates the same magnetic ordering for the electrons in the band. Now consider that the magnetization of the electrons in the band is a simple spiral magnetic ordering

$$
\vec{M}_{0}=M_{0}(\cos q x / a, \sin q x / a, 0) .
$$

The electric current associated with the magnetization is given by

$$
\vec{J}_{M}=\mu c \vec{\nabla} \times \vec{M}_{0}=\frac{\mu c q M_{0}}{a}(0,0, \cos q x / a),
$$

which is a current along $z$ direction. In fact, it is a "global" current along the $z$ direction for a fixed $x$ coordinate. In an insulator, the net electric current with such a configuration must be zero based on Kohn's proof of the insulating property [17], namely, the magnetization current must be counterbalanced by other electric currents. The total electric current contributed from $\vec{j}_{0}$ in the band also vanishes since the lattice mirror symmetry in the $x-y$ plane is not broken in the noncollinear multiferroics $[3,18,19]$ in the absence of external magnetic field. Therefore, the electric current from the magnetic ordering must be counterbalanced by the electric current induced from the spin orbital coupling.

The above analysis can also apply to general noncollinear magnetic ordering structures which induce similar electric currents. From Eq. (4), the cancellation requirement leads to

$$
\mu c \vec{\nabla} \times \vec{M}_{0}+\alpha e \vec{M}_{0} \times \vec{\nabla} V(\vec{r})=\overrightarrow{0} .
$$

By simple algebraic modification and averaging in the total space, Eq. (8) becomes

$$
\frac{\alpha e^{2}}{\mu c}\langle\vec{E}\rangle=\left\langle\frac{\left(\vec{M}_{0} \cdot \vec{E}\right) \vec{M}_{0}}{M_{0}^{2}}\right\rangle+\left\langle\frac{\vec{M}_{0} \times\left(\vec{\nabla} \times \vec{M}_{0}\right)}{M_{0}^{2}}\right\rangle,
$$

where $\langle\ldots\rangle$ takes the space average and $\vec{\nabla} V(\vec{r})=-e \vec{E}(\vec{r})$. The first term in the right side of the above equation usually vanishes when taking the space average for a space modulating spin density. We obtain the total ferroelectricity as

$$
\vec{P}=\frac{\epsilon_{0} \mu c}{\alpha e^{2}}\left\langle\frac{\vec{M}_{0} \times\left(\vec{\nabla} \times \vec{M}_{0}\right)}{M_{0}^{2}}\right\rangle .
$$

Equation (10) is consistent with Eq. (1). However, Eq. (10) provides detailed coupling coefficients that are different from the results normally expected from the Ginzburg-Landau theory. First, the coefficients are inversely proportional to effective spin-orbit coupling, which is against intuitive expectation. Second, Eq. (10) predicts that saturated value of ferroelectricity does not depend on the amplitude of the magnetization. Therefore, it suggests that increasing large magnetization will not dramatically increase ferroelectricity, which is against that normally expected from the simple Ginzburg-Landau theory. Finally, in Eq. (10), there is only one free parameter, the effective spin orbital coupling. The fact of the existence of only one free parameter makes relatively easier to test the new mechanism experimentally.

There are two important issues regarding of the above results. First, it is clear that the above counterintuitive dependence of the polarization on the spin-orbit coupling parameter and the magnitude of magnetization can not be correct for arbitrary small values of these parameters. Therefore, what is the limitation of this mechanism based on the current cancellation? To answer this question, we have to compare the energy saved from the current cancellation and the energy cost from the polarization. When a polarization is developed, there is an energy paid for the deformation of the lattice. This energy cost $E_{\text {cost }}$ for a small polarization is naturally expected to be

$$
E_{\text {cost }}=\frac{1}{2} \lambda \vec{P}^{2},
$$

where $\lambda$ depends on the detailed lattice structures. The energy saved in the current cancellation for the electrons, $E_{\text {save }}$, should be proportional to $\vec{J}_{M}^{2}$. From the simple model considered in the Letter, we can also easily show that

$$
E_{\mathrm{save}}=\frac{m^{*} \mu^{2} c^{2}}{2 e^{2}}\left(\vec{\nabla} \times \vec{M}_{0}\right)^{2} .
$$

In order to favor the current cancellation mechanism, we must have $E_{\text {save }}>E_{\text {cost }}$, which leads to the following criteria for the mechanism of the current cancellation by plugging Eq. (10),

$$
\alpha M_{0}>\sqrt{\frac{\epsilon_{0}^{2} \lambda}{m^{*} e^{2}}} .
$$


This criteria is satisfied in the spiral magnets such as $\mathrm{TbMnO}_{3}$ as we will show later.

Second, a careful reader may notice that there is a critical flaw in the above derivation for a simple band picture: for any magnetic ordering of the localized spins of atoms, the electrons in a fully filled band do not have magnetization response regardless of the strength of spin exchange coupling between them. Namely, $\vec{M}_{0}=\overrightarrow{0}$ for any magnetic order $\vec{M}$ of the localized spins of atoms. Thus the cancellation of the current in Eq. (8) does not exist. This observation leads to two important predictions of the Letter: (i) in order to generate magnetoelectric coupling through the current cancellation, the multiferroics cannot be an conventional insulator; (ii) there is no contribution to the ferroelectricity from the completely filled bands. The contribution only comes from the band which is partially filled. These results are remarkably different from the physics in the conventional ferroelectric materials where the ferroelectricity originates from the instability of lattices and all of the electron bands contribute [20].

There are two possible interactions that can cause the multiferroics to be an insulator with a partially filled band. The first is the strong electron-electron interaction. In this case, the multiferroics is a Mott insulator. The second is the strong spin exchange coupling between the electrons on the band and the localized spin moments. Both mechanisms can split a band into two or more subbands. The simple picture in Fig. 1 shows the splitting of the band into two. If both of the two new subbands are fully occupied, no electric current will be generated by the magnetic ordering of the localized spins, because $\vec{M}_{0}=0$. As a matter of fact, one can picture the physics as the magnetization and electric currents are exactly the opposite in direction in

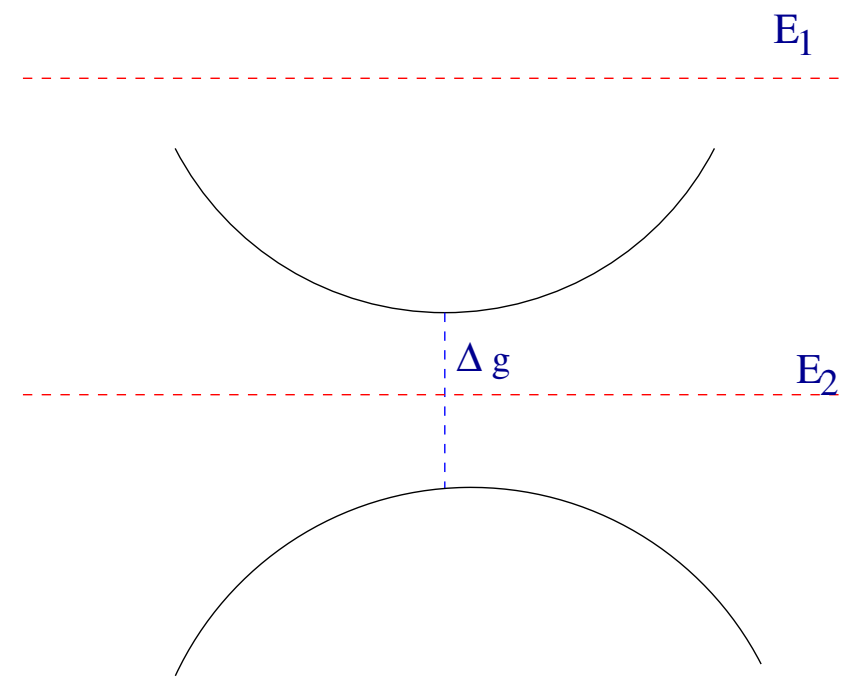

FIG. 1 (color online). A sketch of two bands which are split from one spin degenerate band. $\Delta_{g}$ defines the band gap. When the chemical potential at $E_{1}$, both bands are fully filled. When the chemical potential at $E_{2}$, it is an unconventional band or Mott insulator which can generate ferroelectricity. two new subbands. However, if a gap exists between the two new subbands, and if the lower energy subband is completely filled but the upper energy subband is empty. The spacial curl of the magnetic ordering parameter is a real electric current in the insulator which needs to be counterbalanced by the electric current from the spin orbital coupling.

Now we quantitatively discuss the ferroelectricity predicted by Eq. (10). We rewrite $\alpha=\frac{1}{2 m \Delta_{s}}$ where $\Delta_{s}$ can be viewed as the effective spin-orbit gap, an analogy of the spin-orbit gap in semicondutor [21] . For the spiral magnetic structure in Eq. (6), if we choose reasonable parameters with $\mathrm{g}$ factor to be two, the lattice constant $a=1 \mathrm{~nm}$ and $\Delta_{s}$ with an unit of $\mathrm{eV}$, we obtain

$$
P_{y}=0.88 q \Delta_{s}\left[\mu \mathrm{C} /\left(\mathrm{cm}^{2} \mathrm{eV}\right)\right]
$$

In experiments $[18,19]$ for the spiral magnets such as $\mathrm{TbMnO}_{3}$ and $\mathrm{DyMnO}_{3}, P_{y} \sim 0.1 \mu \mathrm{C} / \mathrm{cm}^{2}$ for $\mathrm{TbMnO}_{3}$, $P_{y} \sim 0.2 \mu \mathrm{C} / \mathrm{cm}^{2}$ for $\mathrm{DyMnO}_{3}$, and the spiral wave vector $q \sim 0.3 \times 2 \pi$ [3]. Consider the fact that theoretical values are generally larger than experimental measurements due to the existence of disorder, Eq. (14) predicts that the $\Delta_{s}$ is in the order of a few $0.1 \mathrm{eV}$ in the spiral multiferroics. This prediction can be tested by future experiments or numerical calculations.

To show that the condition, Eq. (13), is really satisfied in the spiral magnets, we can estimate the cost energy $E_{\text {cost }}$ and the saving energy $E_{\text {save }}$. Taking the polarization value $P_{y} \sim 0.1 \mu \mathrm{C} / \mathrm{cm}^{2}$ observed in $\mathrm{TbMnO}_{3}$ [3], we can estimate the effective lattice shift, $\delta y=P_{y} V_{n} / e^{*}$, where $V_{n}$ is the volume of one unit cell and $e^{*}=N_{\text {eff }} e$ is the effective charge in one unit cell $[9,12]$. The cost energy $E_{\text {cost }}=$ $\frac{1}{2} \kappa \delta_{y}^{2}$, where $\kappa$ is determined by lattice structures. For $\mathrm{TbMnO}_{3}, \kappa \sim 1 \mathrm{eV} / \AA^{2}$ and $V_{n}=2.3 \times 10^{2} \AA^{3}[9,13]$, we have $E_{\text {cost }} \sim\left(\frac{0.014}{N_{\text {eff }}}\right)^{2} \mathrm{eV}$. Let us assume that the number of effective spin of electrons in one unit cell $M_{0}=S_{\text {eff }}(\hbar)$, $m^{*}=b m$ ( $m$ electron mass) and $g=2$, we obtain, $E_{\text {save }} \sim$ $0.35 b S_{\text {eff }}^{2} \mathrm{eV}$ for $q \sim 2 \pi \times 0.3$ in $\mathrm{TbMnO}_{3}$. Comparing $E_{\text {cost }}$ and $E_{\text {save }}$, we see that the Eq. (13) can be satisfied for $S_{\text {eff }}>0.02$. It is important to note that the $S_{\text {eff }}$ is not the total effective spin of magnetized atoms measured in experiments and it is always less than one since there is no contribution from completely filled bands.

Although the condition, Eq. (13), posts a limitation on the current cancellation mechanism, Eq. (14) suggests that the value of ferroelectricity can still grow largely as $\Delta_{s}$ increases. However, in the electric current cancellation mechanism, there is an additional limitation on the value of the polarization. The ferroelectricity is limited by a natural energy scale, the energy gap $\Delta_{g}$ in the insulator. For a given energy gap $\Delta_{g}$, there is a characteristic length scale give by $l_{c}=\frac{h}{\sqrt{2 m^{*} \Delta_{g}}}$. If there is an internal electric field $\vec{E}$ which is spontaneously generated in the system, in order to maintain the validity of the insulator, the value of 
electric field must satisfy $e|\vec{E}| l_{c}<\Delta_{g}$. This requirement sets the limitation of the value of ferroelectricity that can be generated by the mechanism described in this Letter, i.e.,

$$
|P|<\frac{\epsilon_{0} \Delta_{g}}{e l}=0.72 \sqrt{b \Delta_{g}^{3}}\left[\mu \mathrm{C} /\left(\mathrm{cm}^{2} \mathrm{eV}^{3 / 2}\right)\right]
$$

the energy unit of $\Delta_{g}$ is eV. Equation (15) shows that it is very hard for the ferroelectricity in the multiferroics to be larger than a few $\mu \mathrm{C} / \mathrm{cm}^{2}$ because $\Delta_{g}$ should be in a range of a few $\mathrm{eV}$ at most. This explains why the ferroelectricity measured in the multiferroics is much lower than that in conventional ferroelectric materials. For example, the largest ferroelectricity in the family of $R \mathrm{MnO}_{3}$ is $P \sim$ $0.3 \mu \mathrm{C} / \mathrm{cm}^{2}$ [19] which is measured in $\mathrm{DyMnO}_{3}$, while in conventional ferroelectric materials, such as $\mathrm{BaTiO}_{3}$, $P=26 \mu \mathrm{C} / \mathrm{cm}^{2}[20]$.

In summary, this Letter develops a new mechanism to explain magnetoelectric coupling. With a relative small number of free parameters, the mechanism can estimate the value of ferroelectricity. The free parameters can be independently measured using various experimental techniques. For example, the energy gap in the multiferroics and the spin-orbit coupling strength can be measured in optical absorption spectrum. The prediction of the unconventional band or Mott insulator can be tested via numerical calculations as well. Most importantly, the mechanism sets a general guideline for the search of new multiferroics materials with ferroelectricity of larger value.

The new mechanism also provides a new perspective through which magnetoelectric coupling can be understood: focusing on electronic physics rather than on lattice dynamics. The lattice dynamics has been the key to understand conventional ferroelectricity materials because the development of ferroelectricity is tied to phonon softening [20]. However, in many multiferroics, no clear indication for the phonon softening has been found $[2,22]$. Our new mechanism clearly states that in the multiferroics, it is the electronic properties that are responsible for the magnetoelectric coupling.

It is natural to ask if the mechanism we discussed above is universal to all multiferroics. Although it is not easy to test, we believe that the answer is positive. The analyses in this Letter assume the presence of a single magnetic order in the material. With a single magnetic order, the electric current associated to the magnetic order is easy to be defined. In many multiferroics, the magnetic structures are very complicated and can not be described by a single magnetic order parameter [2,23]. A careful construction of electric current due to magnetization is required. These physics await future investigations. Moreover, it is also interesting to study the connection between the mechanisms based on the DM physics [12,13] and the one proposed in this Letter. In fact, it is possible to understand the inverse DM physics based on the reduction of the electric current. The detailed physics will be reported elsewhere [24].

The author thanks Professor P. Muzikar and Professor G. Giuliani for extremely useful discussion. The author also thanks Dr. A. Bernevig, Dr. H. D. Chen, and Professor S. C. Zhang for their useful comments and discussions. The author also acknowledges Fang Chen and KangJun Seo for discussion. This work was supported by the National Science Foundation under Grant No. PHY-0603759.

[1] Y. Tokura, Science 312, 1481 (2006).

[2] S. W. Cheong and M. Mostovoy, Nat. Mater. 6, 13 (2007).

[3] T. Kimura, T. Goto, H. Shintani, K. Ishizaka, T. Arima, and Y. Tokura, Nature (London) 426, 55 (2003).

[4] N. Hur, S. Park, P. A. Sharma, J. S. Ahn, S. Guha, and S. W. Cheong, Nature (London) 429, 392 (2004).

[5] I. Kigomiya, S. Matsumoto, K. Kohn, Y. Fukuda, T. Shoubu, H. Kimura, Y. Noda, and N. Ikeda, Ferroelectrics 286, 167 (2003).

[6] N. Hur, S. Park, P. A. Sharma, S. Guha, and S. W. Cheong, Phys. Rev. Lett. 93, 107207 (2004).

[7] L.C. Chapon, G. R. Blake, M.J. Gutmann, S. Park, N. Hur, P. G. Radaelli, and S.W. Cheong, Phys. Rev. Lett. 93, 177402 (2004).

[8] A. M. Kadomtseva, S. S. Krotov, Y.F. Popov, and G.P. Vorob'ev, Low Temp. Phys. 32, 709 (2006).

[9] H. Katsura, A. V. Balatsky, and N. Nagaosa, Phys. Rev. Lett. 98, 027203 (2007).

[10] I. Dzyaloshinskii, J. Phys. Chem. Solids 4, 241 (1958).

[11] T. Moriya, Phys. Rev. 120, 91 (1960).

[12] H. Katsura, N. Nagaosa, and A. V. Balatsky, Phys. Rev. Lett. 95, 057205 (2005).

[13] I. A. Sergienko and E. Dagotto, Phys. Rev. B 73, 094434 (2006).

[14] M. Mostovoy, Phys. Rev. Lett. 96, 067601 (2006).

[15] L. D. Landau and E. M. Lifshitz, Quantum Mechanics (Non-Relativistic Theory) (Butterworth-Heinemann, London, 1958).

[16] J. Kubler, Theory of Itinerant Electron Magnetism (Oxford Science, Oxford, 2000).

[17] W. Kohn, Phys. Rev. 133, A171 (1964).

[18] T. Goto, T. Kimura, G. Lawes, A.P. Ramirez, and Y. Tokura, Phys. Rev. Lett. 92, 257201 (2004).

[19] T. Kimura, G. Lawes, and A. P. Ramirez, Phys. Rev. Lett. 94, 137201 (2005).

[20] M.E. Lines and A. M. Glass, Principles and Applications of Ferroelectrics and Related Materials (Clarendon Press, Oxford, 1977).

[21] R. Winkler, Spin-Orbit Coupling Effects in TwoDimensional Electron and Hole Systems (Springer, New York, 2003).

[22] A. Sirenko, S. Park, N. Hur, S.-W. Cheong, C. Ulrich, L. Machtoub, B. Keimer, and G. L. Carr, Proceeding of the March Meeting of APS, Los Angeles, CA (APS, New York, 2005).

[23] C. Fang and J. Hu, arXiv:cond-mat/0702366.

[24] J. Hu, F. Chen, and A. Bernevig, (unpublished). 